\title{
Exact Travelling Wave Solutions for Isothermal Magnetostatic Atmospheres by Fan Subequation Method
}

\author{
Hossein Jafari, ${ }^{1,2}$ Maryam Ghorbani, ${ }^{1}$ \\ and Chaudry Masood Khalique ${ }^{2}$ \\ ${ }^{1}$ Department of Mathematics, Faculty of Mathematical Sciences, University of Mazandaran, \\ P.O. Box 47416-95447, Babolsar, Iran \\ 2 International Institute for Symmetry Analysis and Mathematical Modelling, Department of Mathematical \\ Sciences, North-West University, Mafikeng Campus, Private Bag X2046, Mmabatho 2735, South Africa \\ Correspondence should be addressed to Hossein Jafari, jafari_h@math.com
}

Received 1 September 2012; Revised 14 November 2012; Accepted 15 November 2012

Academic Editor: Lan Xu

Copyright (C) 2012 Hossein Jafari et al. This is an open access article distributed under the Creative Commons Attribution License, which permits unrestricted use, distribution, and reproduction in any medium, provided the original work is properly cited.

\begin{abstract}
The equations of magnetohydrostatic equilibria for a plasma in a gravitational field are investigated analytically. An investigation of a family of isothermal magnetostatic atmospheres with one ignorable coordinate corresponding to a uniform gravitational field in a plane geometry is carried out. These equations transform to a single nonlinear elliptic equation for the magnetic vector potential $u$. This equation depends on an arbitrary function of $u$ that must be specified. With choices of the different arbitrary functions, we obtain analytical solutions of elliptic equation using the Fan subequation method.
\end{abstract}

\section{Introduction}

The equations of magnetostatic equilibria have been used extensively to model the solar magnetic structure [1-4]. An investigation of a family of isothermal magnetostatic atmospheres with one ignorable coordinate corresponding to a uniform gravitational field in a plane geometry is carried out. The force balance consists of the $J \wedge B$ force ( $B$ is the magnetic field induction and $J$ is the electric current density), the gravitational force, and gas pressure gradient force. However, in many models, the temperature distribution is specified a priori and direct reference to the energy equations is eliminated. In solar physics, the equations of magnetostatic have been used to model diverse phenomena, such as the slow evolution stage 
of solar flares, or the magnetostatic support of prominences $[5,6]$. The nonlinear equilibrium problem has been solved in several cases [7-9].

Recently, Fan and Hon [10] developed an algebraic method, belonging to the subequation method to seek more new solutions of nonlinear partial differential equations (NLPDEs) that can be expressed as polynomial in an elementary function which satisfies a more general sub-equation, called Fan sub-equation, than other sub-equations like Riccati equation, auxiliary ordinary equation, elliptic equation, and generalized Riccati equation. As we know, the more general analytical exact solutions of the sub-equation are proposed, the more general corresponding exact solutions of NLPDEs will be obtained. Thus, it is very important how to obtain more new solutions to the sub-equation. Fortunately, the Fan subequation method can construct more general exact solutions to the sub-equation that can capture all the solutions of the Riccati equation, auxiliary ordinary equation, elliptic equation, and generalized Riccati equation. Some works using the Fan's technique are presented in [1, 11-16].

In this paper, we obtain the exact travelling wave solutions for the Liouville and sinh-Poisson equations using the Fan sub-equation method. These two models are special cases of magnetostatic atmospheres model. Also in these cases there is force balance between differents forces.

\section{The Basic Idea of Fan Subequation Method}

In this section, we outline the main steps of Fan sub-equation method [11].

Step 1. For a given nonlinear partial differential equation

$$
N\left(u, u_{t}, u_{x}, u_{t t}, u_{x x}, \ldots\right)=0
$$

we consider its travelling wave solutions $u(x, t)=u(\xi), \xi=x-c t$, then (2.1) is reduced to a nonlinear ordinary differential equation

$$
N\left(u(\xi),-c u^{\prime}(\xi), u^{\prime}(\xi), c^{2} u^{\prime \prime}(\xi), u^{\prime \prime}(\xi), \ldots\right)=0
$$

where a prime denotes the derivative with respect to the variable $\xi$.

Step 2. Expand the solution of (2.2) in the form

$$
u(\xi)=\sum_{i=0}^{n} A_{i} \phi^{i}, \quad A_{n} \neq 0,
$$


where $A_{i}(i=0,1, \ldots, n)$ are constants to be determined later and the new variable $\phi$ satisfies the Fan sub-equation

$$
\phi^{\prime}(\xi)=\epsilon \sqrt{\sum_{j=0}^{4} w_{j} \phi^{j}}
$$

where $\epsilon= \pm 1$ and $w_{j}(j=0, \ldots, 4)$ are constants.

Thus, the derivatives with respect to the variable $\xi$ become the derivatives with respect to the variable $\phi$ as follows:

$$
\frac{d u}{d \xi}=\epsilon \sqrt{\sum_{j=0}^{4} w_{j} \phi^{j}} \frac{d u}{d \phi^{\prime}}, \quad \frac{d^{2} u}{d \xi^{2}}=\frac{1}{2} \sqrt{\sum_{j=1}^{4} j w_{j} \phi^{j-1} \frac{d u}{d \phi}}+\sum_{j=0}^{4} w_{j} \phi^{j} \frac{d^{2} u}{d \phi^{2}}
$$

Step 3. Determine $n$ by substituting (2.3) with (2.4) into (2.2) and balancing the linear term of the highest order with the nonlinear term in (2.2).

Step 4. Substituting (2.3) and (2.4) into (2.2) again and collecting all coefficients of $\phi^{i}(i=$ $0,1,2, \ldots, n)$, then setting these coefficients to zero will give a set of algebraic equations with respect to $A_{i}(i=0,1, \ldots, n)$.

Step 5. Solve these algebraic equations to obtain $A_{i}(i=0,1,2, \ldots, n)$. Substituting these results into (2.3) yields the general form of travelling wave solutions.

Step 6. For each solution to (2.4) which depends on the special conditions chosen for the $w_{0}, w_{1}, w_{2}, w_{3}$, and $w_{4}$, it follows from (2.3) obtained from the above steps that the corresponding exact solution of (2.2) can be constructed.

\section{Basic Equations}

The relevant magnetohydrostatic equations consist of the equilibrium equation

$$
J \wedge B-\rho \nabla \Phi-\nabla P=0
$$

which is coupled with Maxwells equations

$$
J=\frac{\nabla \wedge B}{\mu}, \quad \nabla \cdot B=0
$$

where $P, \rho, \mu$, and $\Phi$ are the gas pressure, the mass density, the magnetic permeability, and the gravitational potential, respectively. It is assumed that the temperature is uniform in space 
and that the plasma is an ideal gas with equation of state $p=\rho R_{0} T_{0}$, where $R_{0}$ is the gas constant and $T_{0}$ is the temperature. Then the magnetic field $B$ can be written as

$$
B=\nabla u \wedge e_{x}+B_{x} e_{x}=\left(B_{x}, \frac{\partial u}{\partial z}, \frac{-\partial u}{\partial y}\right)
$$

The form of (3.3) for $B$ ensures that $\nabla \cdot B=0$ and there is no mono pole or defect structure.

Equation (3.1) requires the pressure and density to be of the form [4]

$$
P(y, z)=P(u) e^{-z / h}, \quad \rho(y, z)=\frac{1}{(g h)} P(u) e^{-z / h}
$$

where $h=R_{0} T_{0} / g$ is the scale height. Substituting (3.2)-(3.4) into (3.1), we obtain

$$
\nabla^{2} u+f(u) e^{-z / h}=0
$$

where

$$
f(u)=\mu \frac{d P}{d u}
$$

Equation (3.6) gives

$$
P(u)=P_{0}+\frac{1}{\mu} \int f(u) d u,
$$

where $P_{0}$ is constant. Substituting (3.7) into (3.4), we obtain

$$
\begin{gathered}
P(y, z)=\left(P_{0}+\frac{1}{\mu} \int f(u) d u\right) e^{-z / h}, \\
\rho(y, z)=\frac{1}{g h}\left(P_{0}+\frac{1}{\mu} \int f(u) d u\right) e^{-z / h} .
\end{gathered}
$$

Using transformation $x_{1}+i x_{2}=e^{-z / l} e^{i y / l},(3.5)$ reduces to

$$
\frac{\partial^{2} u}{\partial x_{1}^{2}}+\frac{\partial^{2} u}{\partial x_{2}^{2}}+l^{2} f(u) e^{(2 / l-1 / h) z}=0
$$

These equations have been given in [2].

\section{Applications of the Fan Subequation Method}

In this section, we will employ the Fan sub-equation method for solving (3.9) for specific forms of the function $f(u)$. 


\subsection{Liouville Equation}

We first consider Liouville equation, which is a special case of (3.9), namely,

$$
u_{x x}+u_{t t}-\alpha^{2} l^{2} e^{-2 u}=0
$$

In order to apply the Fan sub-equation method, we use the wave transformation $u(x, t)=$ $u(\xi), \xi=x-c t$ and transform (4.1) into the form

$$
\left(1+c^{2}\right) u^{\prime \prime}=\alpha^{2} l^{2} e^{-2 u}
$$
equation

We next use the transformation $v=e^{-2 u}$ and obtain the nonlinear ordinary differential

$$
\left(1+c^{2}\right) v v^{\prime \prime}-\left(1+c^{2}\right) v^{\prime 2}+2 \alpha^{2} l^{2} v^{3}=0
$$

Using Step 3 given above, we get $n=2$, therefore the solution of (4.3) can be expressed as

$$
v(\xi)=A_{0}+A_{1} \phi+A_{2} \phi^{2}
$$

Following Step 4, we obtain a system of nonlinear algebraic equations for $A_{0}, A_{1}$, and $A_{2}$ :

$$
\begin{aligned}
& 2 \alpha^{2} l^{2} A_{0}^{3}-\epsilon^{2} A_{1}^{2} w_{0}-c^{2} \epsilon^{2} A_{1}^{2} w_{0}+2 \epsilon^{2} A_{0} w_{0}+2 c^{2} \epsilon^{2} A_{0} A_{2} w_{0} \\
& +\frac{1}{2} \epsilon^{2} A_{0} A_{1} w_{1}+\frac{1}{2} c^{2} \epsilon^{2} A_{0} A_{1} w_{1}=0 \\
& 6 \alpha l^{2} A_{0}^{2} A_{1}-2 \epsilon^{2} A_{1} A_{2} w_{0}-2 c^{2} \epsilon^{2} A_{1} A_{2} w_{0}-\frac{1}{2} \epsilon^{2} A_{1}^{2} w_{1}+3 \epsilon^{2} A_{0} A_{2} w_{1} \\
& +3 c^{2} \epsilon^{2} A_{0} A_{1}^{2}+\epsilon^{2} A_{0} A_{1} w_{2}+c^{2} \epsilon^{2} A_{0} A_{1} w_{2}=0 \\
& 6 \alpha^{2} l^{2} A_{0} A_{1}^{2}+6 \alpha^{2} l^{2} A_{0}^{2} A_{2}-2 \epsilon^{2} A_{2}^{2} w_{0}-\frac{1}{2} \epsilon^{2} A_{1} A_{2} w_{1}-\frac{1}{2} c^{2} \epsilon^{2} A_{1} A_{2} w_{1} \\
& +4 \epsilon^{2} A_{0} A_{2} w_{2}+4 c^{2} \epsilon^{2} A_{0} A_{2} w_{2}+\frac{3}{2} \epsilon^{2} A_{0} A_{1} w_{3}+\frac{3}{2} c^{2} \epsilon^{2} A_{0} A_{1} w_{3}=0 \\
& 2 \alpha^{2} l^{2} A_{1}^{3}+12 \alpha^{2} l^{2} A_{0} A_{1} A_{2}-\epsilon^{2} A_{2}^{2} w_{1}-c^{2} \epsilon^{2} A_{2}^{2} w_{1}+\epsilon^{2} A_{1} A_{2} w_{2}+c^{2} \epsilon^{2} A_{1} A_{2} w_{2} \\
& +\frac{1}{2} \epsilon^{2} A_{1}^{2} w_{3}+\frac{1}{2} c^{2} \epsilon^{2} A_{1}^{2} w_{3}+5 \epsilon^{2} A_{0} A_{2} w_{3}+5 c^{2} \epsilon^{2} A_{0} A_{2} w_{3} \\
& +2 \epsilon^{2} A_{0} A_{1} w_{4}+2 c^{2} \epsilon^{2} A_{0} A_{1} w_{4}=0
\end{aligned}
$$




$$
\begin{aligned}
& 6 \alpha^{2} l^{2} A_{1}^{2} A_{2}+6 \alpha^{2} l^{2} A_{0} A_{2}^{2}+\frac{5}{2} \epsilon^{2} A_{1} A_{2} w_{3}+\frac{5}{2} \epsilon^{2} c^{2} A_{1} A_{2} w_{3}+\epsilon^{2} A_{1}^{2} w_{4} \\
& \quad+c^{2} \epsilon^{2} A_{1}^{2} w_{4}+6 \epsilon^{2} A_{0} A_{2} w_{4}+6 c^{2} \epsilon^{2} A_{0} A_{2} w_{4}=0 \\
& 6 \alpha^{2} l^{2} A_{1} A_{2}^{2}+\epsilon^{2} A_{2}^{2} w_{3}+c^{2} \epsilon^{2} A_{2}^{2} w_{1}+\epsilon^{2} A_{1} A_{2} w_{2}+c^{2} \epsilon^{2} A_{2}^{2} w_{3} \\
& \quad+4 \epsilon^{2} A_{1} A_{2} w_{4}+4 c^{2} \epsilon^{2} A_{1} A_{2} w_{4}=0 \\
& 2 \alpha^{2} l^{2} A_{2}^{3}+2 \epsilon^{2} A_{2}^{2} w_{4}+2 c^{2} \epsilon^{2} A_{2}^{2} w_{4}=0 .
\end{aligned}
$$

Case 1. When $w_{0}=w_{1}=w_{3}=0, w_{2}>0, w_{4}<0,(2.4)$ admits a hyperbolic function solution

$$
\phi=\sqrt{-\frac{w_{2}}{w_{4}}} \operatorname{sech}\left(\sqrt{w_{2}} \xi\right)
$$

Thus (4.4) yields the following new solitary wave solution of (2.1) of bell-type

$$
v_{1}(\xi)=\frac{\left(1+c^{2}\right) w_{2}}{\alpha^{2} l^{2}} \operatorname{sech}^{2}\left(\sqrt{w_{2}} \xi\right)
$$

where $w_{2}>0, w_{4}<0, \alpha \neq 0, l \neq 0$, and $c$ are arbitrary constants. Reverting back to the original variables $x$ and $t$, we obtain the solution of (4.1) in the form

$$
u_{1}(x, t)=-\frac{1}{2} \ln \left[\frac{\left(1+c^{2}\right) w_{2}}{\alpha^{2} l^{2}} \operatorname{sech}^{2}\left\{\sqrt{w_{2}}(x-c t)\right\}\right]
$$

Case 2. When $w_{1}=w_{3}=0, w_{0}=w_{2}^{2} / 4 w_{4}, w_{2}<0, w_{4}>0,(2.4)$ admits two hyperbolic function solutions

$$
\phi= \pm \sqrt{-\frac{w_{2}}{2 w_{4}}} \tanh \left(\sqrt{\frac{-w_{2}}{2}} \xi\right)
$$

and so (4.4) yields one family of solitary travelling wave solutions of (4.1) given by

$$
u_{2}(x, t)=-\frac{1}{2} \ln \left[-\frac{\left(1+c^{2}\right) w_{2}}{2 \alpha^{2} l^{2}}+\frac{\left(1+c^{2}\right) w_{2}}{\alpha^{2} l^{2}} \tanh ^{2}\left(\sqrt{-\frac{w_{2}}{2}}(x-c t)\right)\right],
$$

where $w_{2}<0, w_{4}>0, \alpha \neq 0, l \neq 0$, and $c$ are arbitrary constants.

Case 3. When $w_{0}=w_{1}=0, w_{3}= \pm 2 \sqrt{w_{2} w_{4}}, w_{2}>0, w_{4}>0$, (2.4) has two kinds of exact solutions:

$$
\phi=-\frac{\sqrt{w_{2} w_{4}}}{2 w_{4}} \operatorname{sign}\left(w_{3}\right)\left[1+\tanh \left(\frac{\sqrt{w_{2}}}{2} \xi\right)\right]
$$


and (4.4) yields one family of solitary travelling wave solutions of (4.1) given by

$$
\begin{aligned}
u_{3}(x, t)=-\frac{1}{2} \ln [ & \pm \frac{\left(1+c^{2}\right) w_{2}}{\alpha^{2} l^{2}} \operatorname{sign}\left(w_{3}\right)\left[1+\tanh \left(\frac{\sqrt{w_{2}}}{2}(x-c t)\right)\right] \\
& \left.-\frac{\left(1+c^{2}\right) w_{2}}{4 \alpha^{2} l^{2}}\left[1+\tanh \left(\frac{\sqrt{w_{2}}}{2}(x-c t)\right)\right]^{2}\right]
\end{aligned}
$$

where $w_{2}>0, w_{4}>0, \alpha \neq 0, l \neq 0$, and $c$ are arbitrary constants.

Case 4. When $w_{1}=w_{3}=0,(2.4)$ admits three Jacobian elliptic doubly periodic solutions

$$
\begin{aligned}
& \phi=\sqrt{\frac{-w_{2} k^{2}}{w_{4}\left(2 k^{2}-1\right)}} \text { cn }\left(\sqrt{\frac{w_{2}}{2 k^{2}-1}} \xi, k\right), \quad \text { for } w_{0}=\frac{w_{2}{ }^{2} k^{2}\left(k^{2}-1\right)}{w_{4}\left(2 k^{2}-1\right)^{2}}, w_{2}>0, w_{4}<0, \\
& \phi=\sqrt{\frac{-w_{2}}{w_{4}\left(2-k^{2}\right)}} \operatorname{dn}\left(\sqrt{\frac{w_{2}}{2-k^{2}}} \xi, k\right), \quad \text { for } w_{0}=\frac{w_{2}^{2}\left(1-k^{2}\right)}{w_{4}\left(k^{2}-2\right)^{2}}, w_{2}>0, w_{4}<0, \\
& \phi= \pm \sqrt{\frac{-w_{2} k^{2}}{w_{4}\left(k^{2}+1\right)}} \operatorname{sn}\left(\sqrt{\frac{-w_{2}}{k^{2}+1}} \xi, k\right), \quad \text { for } w_{0}=\frac{w_{2}{ }^{2} k^{2}}{w_{4}\left(k^{2}+1\right)^{2}}, w_{2}<0, w_{4}>0,
\end{aligned}
$$

and (4.4), respectively, yields two families of Jacobian elliptic doubly periodic wave solutions

$$
u_{4}(x, t)=-\frac{1}{2} \ln \left[-\frac{\left(1+c^{2}\right) w_{2}}{2 \alpha^{2} l^{2}}+\frac{\left(1+c^{2}\right) w_{2}\left(2 k^{2}-1\right)}{4 \alpha^{2} l^{2}\left(k^{2}-1\right)} \mathrm{cn}^{2}\left(\sqrt{\frac{w_{2}}{2 k^{2}-1}}(x-c t), k\right)\right]
$$

with $w_{2}>0, w_{4}<0, \alpha \neq 0, l \neq 0, k \in(\sqrt{2} / 2,1)$, and $c$ being arbitrary constants. Similarly, from (4.4), respectively, we can obtain two families of Jacobian elliptic doubly periodic wave solutions

$$
u_{5}(x, t)=-\frac{1}{2} \ln \left[-\frac{\left(1+c^{2}\right) w_{2}}{2 \alpha^{2} \beta^{2}}+\frac{\left(1+c^{2}\right) w_{2}\left(k^{2}-2\right)}{4 \alpha^{2} l^{2}\left(1-k^{2}\right)} \operatorname{dn}^{2}\left(\sqrt{\frac{w_{2}}{2-k^{2}}}(x-c t), k\right)\right]
$$

with $w_{2}>0, w_{4}<0, \quad \alpha \neq 0, \quad l \neq 0, k \in(0,1)$, and $c$ being arbitrary constants. Similarly, from (4.4), respectively, we can obtain two families of Jacobian elliptic doubly periodic wave solutions

$$
u_{6}(x, t)=-\frac{1}{2} \ln \left[-\frac{\left(1+c^{2}\right) w_{2}}{2 \alpha^{2} l^{2}}+\frac{\left(1+c^{2}\right) w_{2}\left(k^{2}+1\right)}{4 \alpha^{2} l^{2}} \operatorname{sn}^{2}\left(\sqrt{-\frac{w_{2}}{k^{2}+1}}(x-c t), k\right)\right]
$$

with $w_{2}<0, w_{4}>0, \alpha \neq 0, l \neq 0, k \in(0,1)$, and $c$ being arbitrary constants. 


\subsection{The sinh-Poisson Equation}

Secondly, we consider sinh-Poisson equation which plays an important role in soliton model with BPS Bound. Also, this equation is a special case of (3.9) and is given by

$$
u_{x x}+u_{t t}=\beta^{2} \sinh (u)
$$

In order to apply the Fan sub-equation method, we use the wave transformation $\xi=x-c t$ and convert (4.17) into the form

$$
\left(1+c^{2}\right) u^{\prime \prime}=\beta^{2} \sinh (u)
$$

We next use the transformation $v=e^{u}$ and obtain the equation

$$
2\left(1+c^{2}\right) v v^{\prime \prime}-2\left(1+c^{2}\right) v^{\prime 2}-\beta^{2}\left(v^{3}-v\right)=0
$$

Applying Step 3, we get $n=2$, therefore the solution of (4.19) can be expressed as

$$
v(\xi)=A_{0}+A_{1} \phi+A_{2} \phi^{2} .
$$

Then using Step 4, we obtain a system of nonlinear algebraic equations for $A_{0}, A_{1}$, and $A_{2}$ :

$$
\begin{aligned}
& -l^{2} A_{0}^{3}-2 \epsilon^{2} A_{1}^{2} w_{0}-2 c^{2} \epsilon^{2} A_{1}^{2} w_{0}+4 \epsilon^{2} A_{0} A_{2} w_{0}+4 c^{2} \epsilon^{2} A_{0} A_{2} w_{0} \\
& +\epsilon^{2} A_{0} A_{1} w_{1}+c^{2} \epsilon^{2} A_{0} A_{1} w_{1}=0, \\
& -3 l^{2} A_{0}^{2} A_{1}-4 \epsilon^{2} A_{1} A_{2} w_{0}-4 c^{2} \epsilon^{2} A_{1} A_{2} w_{0}-\epsilon^{2} A_{1}^{2} w_{1}-c^{2} \epsilon^{2} A_{1}^{2} w_{1}+6 \epsilon^{2} A_{0} A_{2} w_{1} \\
& +6 c^{2} \epsilon^{2} A_{0} A_{2} w_{1}+2 \epsilon^{2} A_{0} A_{1} w_{2}+2 c^{2} \epsilon^{2} A_{0} A_{1} w_{2}=0, \\
& -3 l^{2} A_{0} A_{1}{ }^{2}-3 l^{2} A_{0}{ }^{2} A_{2}-4 \epsilon^{2} A_{2}^{2} w_{0}-4 c^{2} \epsilon^{2} A_{2}^{2} w_{0}-\epsilon^{2} A_{1} A_{2} w_{1}-c^{2} \epsilon^{2} A_{1} A_{2} w_{1} \\
& +8 \epsilon^{2} A_{0} A_{2} w_{2}+8 c^{2} \epsilon^{2} A_{0} A_{2} w_{2}+3 \epsilon^{2} A_{0} A_{1} w_{3}+3 c^{2} \epsilon^{2} A_{0} A_{1} w_{3}=0, \\
& -l^{2} A_{1}^{3}-6 l^{2} A_{0} A_{1} A_{2}-2 \epsilon^{2} A_{2}^{2} w_{1}-2 c^{2} \epsilon^{2} A_{2}^{2} w_{1}+2 \epsilon^{2} A_{1} A_{2} w_{2}+2 c^{2} \epsilon^{2} A_{1} A_{2} w_{2} \\
& +\epsilon^{2} A_{1}^{2} w_{3}+c^{2} \epsilon^{2} A_{1}^{2} w_{3}+10 \epsilon^{2} A_{0} A_{2} w_{3}+10 c^{2} \epsilon^{2} A_{0} A_{2} w_{3} \\
& +4 \epsilon^{2} A_{0} A_{1} w_{4}+4 c^{2} \epsilon^{2} A_{0} A_{1} w_{4}=0, \\
& -3 l^{2} A_{1}^{2} A_{2}-3 l^{2} A_{0} A_{2}^{2}+5 \epsilon^{2} A_{1} A_{2} w_{3}+5 c^{2} \epsilon^{2} A_{1} A_{2} w_{3}+2 \epsilon^{2} A_{1}^{2} w_{4}+2 c^{2} \epsilon^{2} A_{1}^{2} w_{4} \\
& +12 \epsilon^{2} A_{0} A_{2} w_{4}+12 c^{2} \epsilon^{2} A_{0} A_{2} w_{4}=0, \\
& -3 l^{2} A_{1} A_{2}{ }^{2}+2 \epsilon^{2} A_{2}{ }^{2} w_{3}+2 c^{2} \epsilon^{2} A_{2}{ }^{2} w_{3}+8 \epsilon^{2} A_{1} A_{2} w_{4}+8 c^{2} \epsilon^{2} A_{1} A_{2} w_{4}=0, \\
& -l^{2} A_{2}{ }^{3}+4 \epsilon^{2} A_{2}^{2} w_{4}+4 c^{2} \epsilon^{2} A_{2}^{2} w_{4}=0 \text {. }
\end{aligned}
$$


Case 1. When $w_{0}=w_{1}=w_{3}=0, w_{2}>0, w_{4}<0,(2.4)$ admits a hyperbolic function solution

$$
\phi=\sqrt{-\frac{w_{2}}{w_{4}}} \operatorname{sech}\left(\sqrt{w_{2}} \xi\right)
$$

and (4.20) yields the following new solitary wave solution of (4.17) of bell-type

$$
u_{1}(x, t)=\ln \left[-\frac{4\left(1+c^{2}\right) w_{2}}{l^{2}} \operatorname{sech}^{2}\left(\sqrt{w_{2}}(x-c t)\right)\right]
$$

where $w_{2}>0, w_{4}<0, l \neq 0$, and $c$ are arbitrary constants.

Case 2. When $w_{1}=w_{3}=0, w_{0}=w_{2}^{2} / 4 w_{4}, w_{2}<0, w_{4}>0$, (2.4) admits two hyperbolic function solutions

$$
\phi= \pm \sqrt{-\frac{w_{2}}{2 w_{4}}} \tanh \left(\sqrt{\frac{-w_{2}}{2}} \xi\right)
$$

and (4.20) yields one family of solitary travelling wave solutions of (4.17) given by

$$
u_{2}(x, t)=\ln \left[\frac{2\left(1+c^{2}\right) w_{2}}{l^{2}}-\frac{2\left(1+c^{2}\right) w_{2}}{l^{2}} \tanh ^{2}\left(\sqrt{-\frac{w_{2}}{2}}(x-c t)\right)\right]
$$

where $w_{2}<0, w_{4}>0, l \neq 0$, and $c$ are arbitrary constants.

Case 3. When $w_{0}=w_{1}=0, w_{3}= \pm 2 \sqrt{w_{2} w_{4}}, w_{2}>0, w_{4}>0$, (2.4) has two kinds of exact solutions

$$
\phi=-\frac{\sqrt{w_{2} w_{4}}}{2 w_{4}} \operatorname{sign}\left(w_{3}\right)\left[1+\tanh \left(\frac{\sqrt{w_{2}}}{2} \xi\right)\right]
$$

and (4.20) yields one family of solitary travelling wave solutions solitary travelling wave solutions of (4.17) given by

$$
\begin{aligned}
u_{3}(x, t)=\ln & {\left[ \pm \frac{2\left(1+c^{2}\right) w_{2}}{l^{2}} \operatorname{sign}\left(w_{3}\right)\left[1+\tanh \left(\frac{\sqrt{w_{2}}}{2}(x-c t)\right)\right]\right.} \\
& \left.-\frac{\left(1+c^{2}\right) w_{2}}{l^{2}}\left[1+\tanh \left(\frac{\sqrt{w_{2}}}{2}(x-c t)\right)\right]^{2}\right]
\end{aligned}
$$

where $w_{2}>0, w_{4}>0, l \neq 0$ and $c$ are arbitrary constants. 
Case 4. When $w_{1}=w_{3}=0,(2.4)$ admits three Jacobian elliptic doubly periodic solutions

$$
\begin{aligned}
& \phi=\sqrt{\frac{-w_{2} k^{2}}{w_{4}\left(2 k^{2}-1\right)}} \text { cn }\left(\sqrt{\frac{w_{2}}{2 k^{2}-1}} \xi, k\right), \quad \text { for } w_{0}=\frac{w_{2}{ }^{2} k^{2}\left(k^{2}-1\right)}{w_{4}\left(2 k^{2}-1\right)^{2}}, w_{2}>0, w_{4}<0, \\
& \phi=\sqrt{\frac{-w_{2}}{w_{4}\left(2-k^{2}\right)}} \operatorname{dn}\left(\sqrt{\frac{w_{2}}{2-k^{2}}} \xi, k\right), \quad \text { for } w_{0}=\frac{w_{2}^{2}\left(1-k^{2}\right)}{w_{4}\left(k^{2}-2\right)^{2}}, w_{2}>0, w_{4}<0, \\
& \phi= \pm \sqrt{\frac{-w_{2} k^{2}}{w_{4}\left(k^{2}+1\right)}} \operatorname{sn}\left(\sqrt{\frac{-w_{2}}{k^{2}+1}} \xi, k\right), \quad \text { for } w_{0}=\frac{w_{2}^{2} k^{2}}{w_{4}\left(k^{2}+1\right)^{2}}, w_{2}<0, w_{4}>0,
\end{aligned}
$$

and (4.20), respectively, yields two families of Jacobian elliptic doubly periodic wave solutions

$$
u_{4}(x, t)=\ln \left[\frac{2\left(1+c^{2}\right) w_{2}}{l^{2}}+\frac{2\left(1+c^{2}\right)\left(2 k^{2}-1\right) w_{2}}{l^{2}\left(k^{2}-1\right)} \mathrm{cn}^{2}\left(\sqrt{\frac{w_{2}}{2 k^{2}-1}}(x-c t), k\right)\right]
$$

with $w_{2}>0, w_{4}<0, l \neq 0, k \in(\sqrt{2} / 2,1)$, and $c$ being arbitrary constants. Similarly, from (4.20), respectively, we can obtain two families of Jacobian elliptic doubly periodic wave solutions

$$
u_{5}(x, t)=\ln \left[\frac{2\left(1+c^{2}\right) w_{2}}{l^{2}}-\frac{2\left(1+c^{2}\right) w_{2}\left(2-k^{2}\right)}{l^{2}\left(1-k^{2}\right)} \operatorname{dn}^{2}\left(\sqrt{\frac{w_{2}}{2-k^{2}}}(x-c t), k\right)\right]
$$

with $w_{2}>0, w_{4}<0, \quad \alpha \neq 0, l \neq 0, k \in(0,1)$, and $c$ being arbitrary constants. Likewise, from (4.20), respectively, we can get two families of Jacobian elliptic doubly periodic wave solutions

$$
u_{6}(x, t)=\ln \left[\frac{2\left(1+c^{2}\right) w_{2}}{l^{2}}-\frac{2\left(1+c^{2}\right) w_{2}\left(k^{2}+1\right)}{l^{2}} \operatorname{sn}^{2}\left(\sqrt{-\frac{w_{2}}{k^{2}+1}}(x-c t), k\right)\right]
$$

with $w_{2}<0, w_{4}>0, \alpha \neq 0, l \neq 0, k \in(0,1)$, and $c$ being arbitrary constants.

\section{Concluding Remarks}

In this paper, the Fan sub-equation method has been successfully used to obtain some exact travelling wave solutions for the Liouville and sinh-Poisson equations. These exact solutions include the hyperbolic function solutions, trigonometric function solutions. When the parameters are taken as special values, the solitary wave solutions are derived from the hyperbolic function solutions. Thus, this study shows that the Fan sub-equation method is quite efficient and practically well suited for use in finding exact solutions for nonlinear partial differential equations. The reliability of the method and the reduction in the size of computational domain give this method a wider applicability. 


\section{References}

[1] A. H. Khater, M. A. El-Attary, M. F. El-Sabbagh, and D. K. Callebaut, "Two-dimensional magnetohydrodynamic equilibria," Astrophysics and Space Science, vol. 149, no. 2, pp. 217-223, 1988.

[2] A. H. Khater, D. K. Callebaut, and O. H. El-Kalaawy, "Bäcklund transformations and exact solutions for a nonlinear elliptic equation modelling isothermal magnetostatic atmosphere," IMA Journal of Applied Mathematics, vol. 65, no. 1, pp. 97-108, 2000.

[3] I. Lerche and B. C. Low, "Some nonlinear problems in astrophysics," Physica D. Nonlinear Phenomena, vol. 4, no. 3, pp. 293-318, 1981/82.

[4] B. C. Low, "Evolving force-free magnetic elds. I-the development of the pre are stage," The Astrophysical Journal, vol. 212, pp. 234-242, 1977.

[5] X.-H. Wu and J.-H. He, "Solitary solutions, periodic solutions and compacton-like solutions using the Exp-function method," Computers E Mathematics with Applications, vol. 54, no. 7-8, pp. 966-986, 2007.

[6] W. Zwingmann, "Theoretical study of onset conditions for solar eruptive processes," Solar Physics, vol. 111, pp. 309-331, 1987.

[7] I. Lerche and B. C. Low, "On the equilibrium of a cylindrical plasma supported horizontally by magneticelds in uniform gravity," Solar Physics, vol. 67, pp. 229-243, 1980.

[8] G. M. Webb, "Isothermal magnetostatic atmospheres. II-similarity solutions with current proportional to the magnetic potential cubed," The Astrophysical Journal, vol. 327, pp. 933-949, 1988.

[9] G. M. Webb and G. P. Zank, "Application of the sine-Poisson equation in solar magnetostatics," Solar Physics, vol. 127, pp. 229-252, 1990.

[10] E. G. Fan and Y. C. Hon, "A series of travelling wave solutions for two variant Boussinesq equations in shallow water waves," Chaos, Solitons E Fractals, vol. 15, no. 3, pp. 559-566, 2003.

[11] D. Feng and K. Li, "Exact traveling wave solutions for a generalized Hirota-Satsuma coupled KdV equation by Fan sub-equation method," Physics Letters A, vol. 375, no. 23, pp. 2201-2210, 2011.

[12] S. A. El-Wakil and M. A. Abdou, "The extended Fan sub-equation method and its applications for a class of nonlinear evolution equations," Chaos, Solitons and Fractals, vol. 36, no. 2, pp. 343-353, 2008.

[13] E. Yomba, "The modified extended Fan sub-equation method and its application to the $(2+1)$ dimensional Broer-Kaup-Kupershmidt equation," Chaos, Solitons and Fractals, vol. 27, no. 1, pp. 187196, 2006.

[14] E. Yomba, "The extended Fan's sub-equation method and its application to KdV-MKdV, BKK and variant Boussinesq equations," Physics Letters A, vol. 336, no. 6, pp. 463-476, 2005.

[15] S. Zhang and H.-Q. Zhang, "Fan sub-equation method for Wick-type stochastic partial differential equations," Physics Letters A, vol. 374, no. 41, pp. 4180-4187, 2010.

[16] S. Zhang and T. Xia, "A further improved extended Fan sub-equation method and its application to the $(3+1)$-dimensional Kadomstev-Petviashvili equation," Physics Letters A, vol. 356, no. 2, pp. 119-123, 2006. 


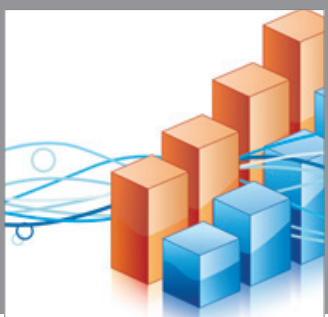

Advances in

Operations Research

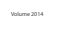

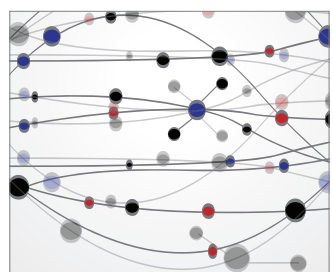

\section{The Scientific} World Journal
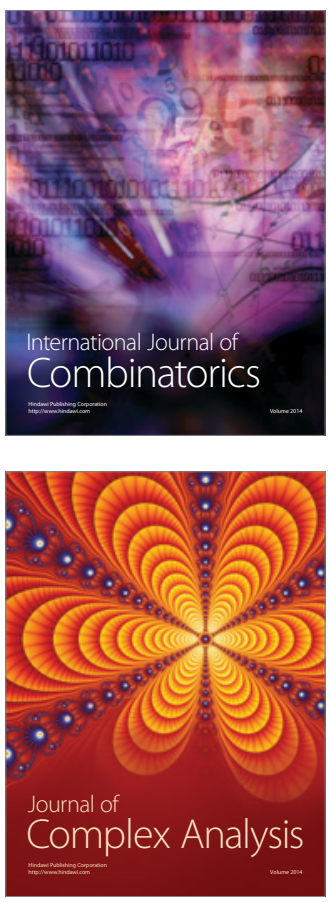

International Journal of

Mathematics and

Mathematical

Sciences
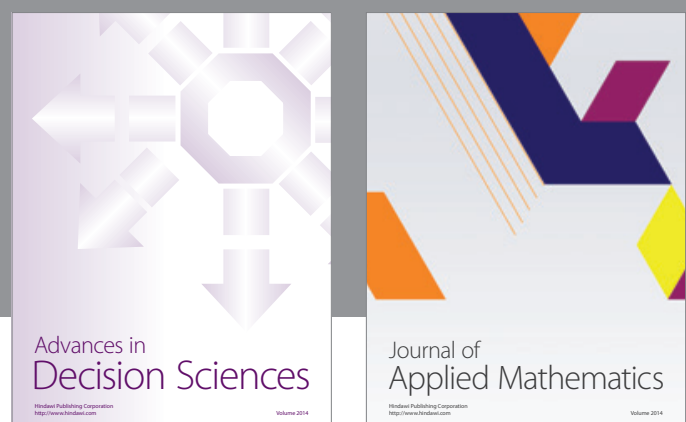

Journal of

Applied Mathematics
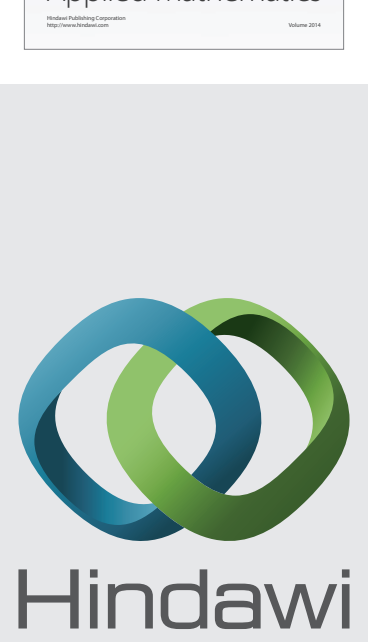

Submit your manuscripts at http://www.hindawi.com
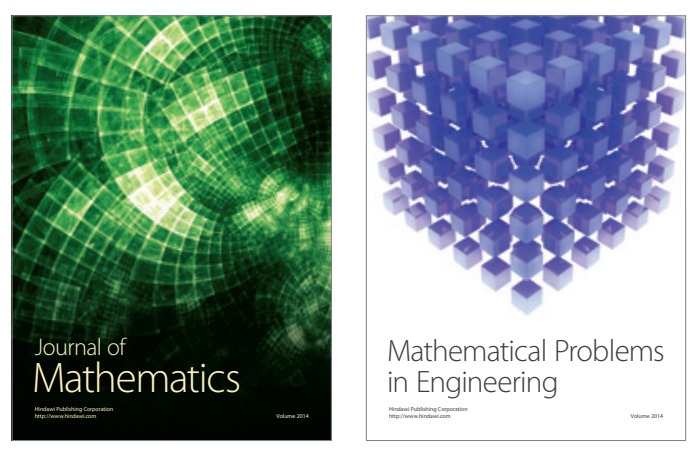

Mathematical Problems in Engineering
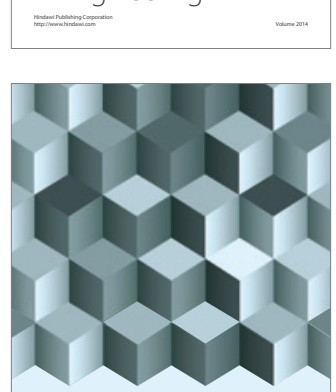

Journal of

Function Spaces
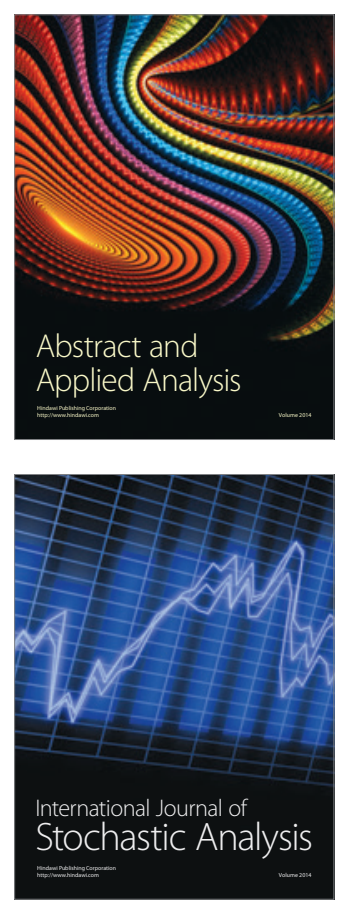

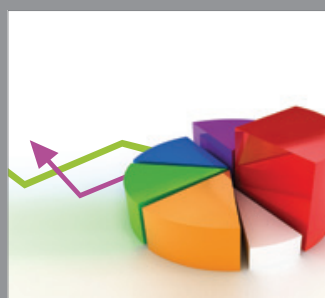

ournal of

Probability and Statistics

Promensencen
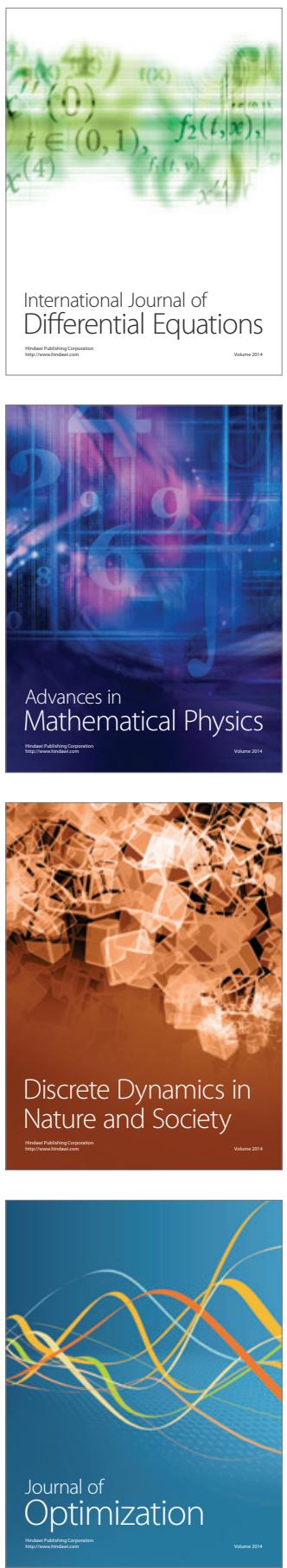